\title{
Validación por expertos del Î́ndice de Distrés Peri-traumático relacionado con la Enfermedad por Coronavirus 2019 para su uso en el Perú.
}

\author{
Expert validation of the COVID-2019 Peri-traumatic Distress Index for use in Peru. \\ Bruno Pedraz-Petrozzi ${ }^{1, a}$, Martín Arévalo-Flores ${ }^{2, b}$, Hever Krüger-Malpartida ${ }^{2, c}$, Victor Anculle-Arauco ${ }^{2, \mathrm{~d}}$
}

\section{RESUMEN}

Objetivo: Efectuar una validación de contenido por expertos del Índice de Distrés Peri-traumático relacionado a COVID-19 (CPDI) para su uso en el Perú. Material y Métodos: El CPDI fue traducido al castellano y luego adaptado en una primera ronda de expertos. En una segunda ronda $(n=13)$, se evaluó la validez del contenido mediante el uso de la V de Aiken (V). Se calculó la V para cada ítem, así como para el instrumento en su conjunto $\left(\mathrm{V}_{\text {тот }}\right)$. Resultados: De los 24 ítems originales, solo un ítem (S7) presentó valores no significativos para validez de contenido, razón por la que fue eliminado. La escala con 23 ítems no presentó alteraciones en el cálculo de la $\mathrm{V}_{\text {TOт }}$. Conclusiones: Sobre la base de su validez de contenido, el CPDI en castellano es adecuado para su aplicación en la población peruana. Quedan por definirse las características psicométricas así como la modificación propuesta del instrumento, mediante estudios cuantitativos en torno a la validez del constructo.

PALABRAS CLAVE: COVID-19, pandemias, estudio de validación, estrés emocional, salud mental.

\section{SUMMARY}

Objectives: To perform an expert validation study of COVID-19 Peritraumatic Distress Index (CPDI) for its eventual use in Perú. Material and Methods: In a first round by experts, the CPDI was translated into Spanish and then adapted. In a second round, $(\mathrm{n}=13)$, the content validity was evaluated by using Aiken's V $(\mathrm{V})$. The V was calculated for each item, as well as for the whole instrument $\left(\mathrm{V}_{\text {TOT }}\right)$. Results: Of the 24 original items, only one (S7) presented non-significant values for content validity, reason for which it was removed. The final 23 -items scale did not present any alterations for the VTOT calculation Conclusions: On the basis of its adequate content validity, the

Centro de Neurociencias, Centro de Psiquiatría, Universidad Justus-Liebig. Giessen, Alemania.

2 Servicio de Neuropsiquiatría, Hospital Nacional Cayetano Heredia. Lima, Perú.

a Médico. Candidato doctoral (Ph.D.). Contribución del autor: escribió la introducción, materiales y métodos, hizo el análisis estadístico, escribió los resultados y la discusión. ORCID: https://orcid.org/0000-0002-4119971X

b Psiquiatra.Contribución del autor: asesor clínico, consiguió la escala original, corrigió el manuscrito, escribió la introducción. ORCID: https://orcid.org/0000-0001-7849-8839

c Psiquiatra. Contribución del autor: asesor clínico, escribió el resumen, corrigió el manuscrito, escribió la discusión. ORCID: https://orcid.org/0000-0001-7872-9318

d Médico residente de psiquiatría Contribución del autor: escribió los materiales y métodos, contactó a los expertos, recopiló la información. ORCID: https://orcid.org/0000-0001-5996-0039 
Validación por expertos del Índice de Distrés Peri-traumático relacionado con la Enfermedad por Coronavirus 2019.

Spanish version of CPDI is suitable for its application in the Peruvian population. The psychometric characteristics of the instrument, as well as its proposed modification remain to be defined through quantitative studies of the construct validity.

KEYWORDS: COVID-19, Pandemics, validation studies, psychological distress, mental health.

\section{INTRODUCCIÓN}

La enfermedad por infección del SARSCoV-2 (COVID-19) es una entidad viral altamente transmisible y con alta mortalidad en determinados grupos de riesgo (1). Para poder detener el avance de la enfermedad, la Organización Mundial de la Salud (OMS) decretó el estado de emergencia sanitaria a nivel mundial (2). Siguiendo las pautas propuestas por la OMS, muchos gobiernos aplicaron medidas preventivas al contagio, entre ellos el Gobierno del Perú, que decretó el 15 de marzo del 2020 el aislamiento estricto con excepciones básicas y lo terminó el 6 de julio, es decir, más de 16 semanas (2). Posteriormente se fueron flexibilizando las medidas preventivas (3). A consecuencia de ello, la población tuvo un cambio gradual en sus rutinas y actividades de la vida diaria (4). Se considera que las limitaciones para la movilización, problemas económicos, laborales, temor a enfermarse, entre otros generarán un impacto emocional negativo, conllevando consecuencias riesgosas para la salud mental de la población (5).

Para medir este impacto, existen instrumentos en otros países que fueron diseñados durante la pandemia actual y que lo miden en relación a la COVID-19. Un ejemplo de estos instrumentos es el Índice de Distrés Peri-traumático relacionado a coronavirus 2019 (CPDI) (6). El CPDI, validado en China, fue diseñado para evaluar específicamente el impacto emocional de la pandemia por COVID-19 relacionado al estado del ánimo, comportamiento, habilidades cognitivas, ciclo sueño-vigilia y síntomas somáticos (6). Este instrumento fue aplicado en más de 30 provincias chinas y en otros países como en Irán y Brasil $(7,8)$.

El Perú no cuenta actualmente con instrumentos capaces de medir específicamente el impacto emocional relacionado a COVID-19. Debido a la falta de instrumentos específicos para determinar dicho impacto y a los esfuerzos del gobierno peruano por priorizar la atención en salud mental debida a COVID-19 (9), es que se hace necesario poder contar instrumentos como el mencionado CPDI para poder posteriormente intervenir de manera más efectiva en la población general.
Por lo tanto, el principal objetivo de este trabajo es la traducción, adaptación y validación por expertos del CPDI. A partir de esto último, se podrá medir la validez de contenido y el grado de consistencia de los expertos en la valoración de los ítems del CPDI. Adicionalmente, se determinará algunas propiedades psicométricas, para poder evaluar, por ejemplo, si el CPDI debería permanecer en su versión original $o$ en su defecto deben realizarse modificaciones para su aplicación a la población peruana. Validar un instrumento específico para evaluar el impacto emocional relacionado a COVID-19 permitirá que futuros estudios puedan aplicar el instrumento para diferentes grupos poblacionales.

\section{MATERIAL Y MÉTODOS}

El siguiente trabajo es un estudio de validación cualitativa, en donde se tradujo, adaptó y validó el CPDI para su posterior uso en el Perú.

\section{Procedimientos para la traducción y adaptación lingüística del instrumento}

El cuestionario original (COVID-19 Peritraumatic Distress Index) fue publicado en el idioma chino por Qiu et al. y traducido al inglés por los mismos autores (6). El instrumento original consta de 4 dimensiones (D1: Estado de ánimo negativo, D2: Cambios en el comportamiento y en las habilidades cognitivas, D3: Cansancio e hiperreactividad, D4: Somatización) y 24 ítems en total. La tabla 1 muestra el número de dimensiones y el número de ítems por cada dimensión. La versión en inglés del CPDI fue traducida al castellano y re-traducida al inglés para su comparación con la versión original, evitando así posibles discrepancias. Luego de evidenciar que no hubiera discrepancias entre ambos documentos, se envió la versión traducida a una primera comisión de expertos para su evaluación. Esta comisión estuvo conformada por tres psiquiatras con amplia experiencia clínica. Finalmente, la primera ronda de expertos evaluó las traducciones e hicieron las observaciones correspondientes para producir una versión adaptada del CPDI. 


\section{Validación por expertos}

Una vez que se tradujo y realizó las observaciones señaladas por los expertos, se envió esta nueva versión a una segunda ronda de expertos $(n=13)$ con la finalidad de calcular la validez del contenido entre los expertos. En este caso, los convocados fueron psiquiatras con experiencia clínica, sobretodo con experiencia para la intervención de personas con ansiedad, depresión y trauma relacionado a evento. Los psiquiatras includos para la evaluación fueron profesionales con conocimientos del manejo de pacientes en situaciones de emergencia como tambien de pacientes ambulatorios.

\section{Validez del contenido y analisis de los datos}

Con respecto a la validez del contenido de un instrumento, esta se define como el grado en el que el contenido del instrumento refleja adecuadamente lo que se propone medir y es, por ende, representativo para dicho contenido (10). Para ello, se calculó la validez del contenido usando la metodología de Aiken ( $\mathrm{V}$ de Aiken, $\mathrm{V}=0$ : total desacuerdo, $\mathrm{V}=1$ : total acuerdo, $V=0,5$ : indecisión) (11) que evalúa el número de acuerdos entre los expertos sobre el instrumento. Para poder medir el acuerdo entre los jueces, por cada ítem se formularon dos preguntas relacionadas con la pertinencia del ítem, incluyendo a su vez elementos tipo Likert de 5 categorías ("totalmente en desacuerdo", "en desacuerdo", "neutral", "de acuerdo" y "totalmente de acuerdo").

A partir de la información obtenida por los jueces sobre ambos elementos, se calculó la V de Aiken $(12,13)$. Este coeficiente se calculó para cada ítem, así como para el conjunto total de ítems que conforman el intstrumento $\left(\mathrm{V}_{\text {TOT }}\right)$. Se calculó los intervalos de confianza correspondientes al 95\% (IC95) $(12,14)$, para estimar los parámetros poblacionales para el valor de $\mathrm{V}\left(\mathrm{V}_{\mathrm{p}}\right)$. Se definió la hipótesis nula como la probabilidad de que el $\mathrm{V}_{\mathrm{p}}$ tome el valor igual o menor a 0,5 (12). Para aceptar la hipótesis nula, el IC95 debe incluir el valor 0,5 dentro de su rango de valores, dando así la posibilidad de que $\mathrm{V}_{\mathrm{p}}=0,5$. A continuación, para decidir si el ítem permanecía en el instrumento, se evaluó los IC95 y se verificó que la hipótesis nula no se cumpliera. En caso de que se cumpliese la hipótesis nula, el ítem debía de ser descartado.

Finalmente, para el análisis de los datos según la metodología de Aiken, este fue realizado en el programa Microsoft Excel 2019 para Macintosh, siguiendo las fórmulas de Aiken $(12,14,15)$ y las modificaciones por Penfield y Giacobbi (12).

\section{RESULTADOS}

\section{Fase de traducción, re-traducción y adaptación cultural}

Durante la traducción y la re-traducción no se encontró ningún ítem que necesitase alteración. Las preguntas se mantuvieron en el significado de la versión original. En la primera evaluación por el comité de expertos, el lenguaje del CPDI se adaptó a la comprensión de un ciudadano peruano promedio basado en la experiencia de los jueces en su interacción con pacientes y familiares (sin patologías mentales) de diversos estratos socio-económicos, por esto todos los jueces tenían experiencia en la práctica pública y privada. El resultado final de esta fase se presenta en la tabla 2.

\section{Fase de evaluación por jueces para la validación de los ítems}

\section{Validez de los contenidos}

De los ítems originales (S1 a S24), solo el ítem S7 mostró intervalos de confianza que incluyen los valores de la hipótesis nula. Los valores para la $\mathrm{V}$ de Aiken de los ítems restantes se muestran en la tabla 3. Por otro lado, las dimensiones originales mostraron valores significativos para la $\mathrm{V}$ de Aiken. Finalmente, el $\mathrm{V}_{\text {тот }}$ para el CPDI fue de $\mathrm{V}=0,79$, IC95 [0,64; $0,89]$.

Tabla 1. Dimensiones del instrumento CPDI, contenido de cada una de las dimensiones e ítems que representan la dimensión.

\begin{tabular}{cll}
\hline Dimensión & \multicolumn{1}{c}{ Contenido } & \multicolumn{1}{c}{ Ítems que representan la dimensión } \\
\hline D1 & Estado de ánimo negativo & $\mathrm{S} 1, \mathrm{~S} 2, \mathrm{~S} 3, \mathrm{~S} 4, \mathrm{~S} 5$ \\
D2 & Cambios en el comportamiento y en las habilidades cognitivas & $\mathrm{S} 6, \mathrm{~S} 7, \mathrm{~S} 8, \mathrm{~S} 9, \mathrm{~S} 10, \mathrm{~S} 11, \mathrm{~S} 12$ \\
D3 & Cansancio e hiperreactividad & $\mathrm{S} 13, \mathrm{~S} 14, \mathrm{~S} 15, \mathrm{~S} 16, \mathrm{~S} 17, \mathrm{~S} 20, \mathrm{~S} 21$ \\
D4 & Somatización & $\mathrm{S} 18, \mathrm{~S} 19, \mathrm{~S} 22, \mathrm{~S} 23, \mathrm{~S} 24$ \\
\hline
\end{tabular}


Validación por expertos del Índice de Distrés Peri-traumático relacionado con la Enfermedad por Coronavirus 2019.

Tabla 2. 24 ítems que conforman el instrumento original, donde se pueden observar las versiones original, traducida y adaptada.

\begin{tabular}{ll}
\hline Ítem & Versión Original \\
\hline S1 & $\begin{array}{l}\text { Compared to usual, I feel more } \\
\text { nervous and anxious }\end{array}$ \\
S2 & $\begin{array}{l}\text { I feel insecure and bought a lot } \\
\text { of masks, medications, sanitizer, } \\
\text { gloves and/or other home supplies }\end{array}$ \\
& $\begin{array}{l}\text { I can't stop myself from imagining } \\
\text { myself or my family being infected } \\
\text { and feel terrified and anxious about } \\
\text { it }\end{array}$ \\
S3 & $\begin{array}{l}\text { I feel empty and helpless no matter } \\
\text { what I do }\end{array}$ \\
S4
\end{tabular}

S5 patients and their families. I feel sad about them.

I feel helpless and angry about

S6 people around me, governors, and media

S7 I am losing faith in the people around me

I collect information about

S8 COVID-19 all day. Even if it's not necessary, I can't stop myself

I will believe the COVID-19

S9 information from all sources without any evaluation

I would rather believe in negative

S10 news about COVID-19 and be skeptical about the good news

S11 I am constantly sharing news about COVID-19(mostly negative news)

I avoid watching COVID-19 news, since I am too scared to do so

S13 I am more irritable and have frequent conflicts with my family

I feel tired and sometimes even exhausted

Due to feelings of anxiety, my reactions are becoming sluggish.

Traducción y re traducción

Me siento más ansioso y nervioso de lo común.

Me siento inseguro y he estado comprando muchas mascarillas, remedios, gel desinfectante, guantes y/o otros suministros para la casa

No puedo evitar imaginarme que posiblemente mi familia o yo seamos infectados y me siento aterrado y ansioso al pensarlo

Me siento vacío e indefenso sin importar lo que haga

Siento simpatía por los pacientes con COVID-19 y sus familias. Me siento triste por ellos.

Me siento impotente y enojado por la gente al mi alrededor, autoridades, y los medios de comunicación

Estoy perdiendo la fé en la gente de mi entorno

Recopilo información sobre el COVID-19 todo el tiempo. Aunque no sea necesario, no puedo evitarlo

Creo en todas las fuentes con información sobre el COVID-19 sin evaluarlas previamente

Prefiero creer en las noticias negativas sobre el COVID-19 y tener una opinión más escéptica sobre las noticias buenas

Estoy constantemente compartiendo noticias sobre el COVID-19 (mayormente información negativa)

Evito ver noticias sobre el COVID-19 por el miedo que me genera

Me siento más irritable y estoy teniendo conflictos frecuentes con mi familia

Me siento cansado y a veces hasta exhausto

Debido a la ansiedad, mis reacciones se están volviendo más lentas
Adaptación cultural por expertos

Me siento más ansioso y nervioso que lo usual

Me siento inseguro y he estado comprando muchas mascarillas, remedios, gel desinfectante, guantes $\mathrm{y} / \mathrm{u}$ otros productos para la casa.

No puedo dejar de imaginar que posiblemente mi familia o yo seamos infectados y me siento aterrado y ansioso al pensarlo.

No importa lo que haga, me siento vacio e indefenso.

Siento compasión por los pacientes con COVID-19 y sus familias. Me siento triste por ellos.

Me siento impotente y enojado con la gente a mi alrededor, autoridades y los medios de comunicación

Estoy perdiendo la fe en la gente de mi entorno.

Estoy pendiente de la información sobre el COVID-19 todo el tiempo. Incluso si no es necesario. No puedo evitarlo.

Creo en todas las fuentes con información sobre el COVID-19 sin evaluarlas previamente.

Tiendo a creer en las noticias negativas sobre el COVID-19 y a tener una opinión más escéptica sobre las noticias buenas.

Estoy constantemente compartiendo noticias sobre el COVID-19

(mayormente noticias negativas).

Evito ver noticias sobre el

COVID-19 por el miedo que me genera.

Me siento más irritable y estoy teniendo conflictos frecuentes con mi familia.

Me siento cansado y a veces hasta totalmente sin fuerzas.

Debido a la ansiedad, mis reacciones se están volviendo más lentas.

(Página siguiente) 
Tabla 2. 24 ítems que conforman el instrumento original, donde se pueden observar las versiones original, traducida y adaptada.

\begin{tabular}{|c|c|c|c|}
\hline Ítem & Versión Original & Traducción y re traducción & Adaptación cultural por expertos \\
\hline S16 & I find it hard to concentrate & Me es difícil concentrarme & Me es dificil concentrarme. \\
\hline S17 & I find it hard to make any decisions & Me es difícil tomar decisiones & Me es dificil tomar decisiones. \\
\hline S18 & $\begin{array}{l}\text { During this COVID-19 period, I } \\
\text { often feel dizzy or have back pain } \\
\text { and chest distress }\end{array}$ & $\begin{array}{l}\text { Durante este periodo de } \\
\text { COVID-19, regularmente me } \\
\text { siento mareado o tengo dolor de } \\
\text { espalda y molestia en el pecho }\end{array}$ & $\begin{array}{l}\text { Durante este periodo de COVID-19, } \\
\text { suelo sentirme mareado o tengo } \\
\text { dolor de espalda y/o molestias en el } \\
\text { pecho. }\end{array}$ \\
\hline S19 & $\begin{array}{l}\text { During this COVID-19 period, I } \\
\text { often feel stomach pain, bloating, } \\
\text { and other stomach discomfort }\end{array}$ & $\begin{array}{l}\text { Durante este periodo de } \\
\text { COVID-19, suelo tener dolor } \\
\text { de estomago, hinchazón, y otras } \\
\text { molestias estomacales }\end{array}$ & $\begin{array}{l}\text { Durante este periodo de COVID-19, } \\
\text { suelo tener dolor de estomago, } \\
\text { hinchazón y otras molestias } \\
\text { estomacales. }\end{array}$ \\
\hline $\mathrm{S} 20$ & $\begin{array}{l}\text { I feel uncomfortable when } \\
\text { communicating with others }\end{array}$ & $\begin{array}{l}\text { Me siento incómodo al } \\
\text { comunicarme con otras personas }\end{array}$ & $\begin{array}{l}\text { Me siento incómodo al } \\
\text { comunicarme con otras personas. }\end{array}$ \\
\hline $\mathrm{S} 21$ & Recently, I rarely talk to my family & $\begin{array}{l}\text { Recientemente, rara vez hablo } \\
\text { con mi familia }\end{array}$ & $\begin{array}{l}\text { Estoy hablando menos con } \mathrm{mi} \\
\text { familia. }\end{array}$ \\
\hline S22 & $\begin{array}{l}\text { I cannot sleep well. I always dream } \\
\text { about myself or my family being } \\
\text { infected by COVID-19 }\end{array}$ & $\begin{array}{l}\text { No logro dormir bien. Siempre } \\
\text { sueño que mi familia o yo } \\
\text { estamos infectados con el } \\
\text { COVID-19 }\end{array}$ & $\begin{array}{l}\text { No puedo dormir bien. Sueño que } \\
\text { mi familia o yo estamos infectados } \\
\text { con el COVID-19. }\end{array}$ \\
\hline S23 & I lost my appetite & Perdí mi apetito & He perdido el apetito. \\
\hline S24 & $\begin{array}{l}\text { I have constipation or frequent } \\
\text { urination }\end{array}$ & $\begin{array}{l}\text { Tengo estreñimiento o ganas de } \\
\text { orinar muy seguido }\end{array}$ & $\begin{array}{l}\text { Tengo estreñimiento o ganas de } \\
\text { orinar muy seguido. }\end{array}$ \\
\hline
\end{tabular}

Tabla 3. Validación cualitativa por 13 jueces del instrumento CPDI en su versión 24 ítems (original) y Versión modificada de 23 ítems.

CPDI Original, 24 items CPDI modificada, 23 items

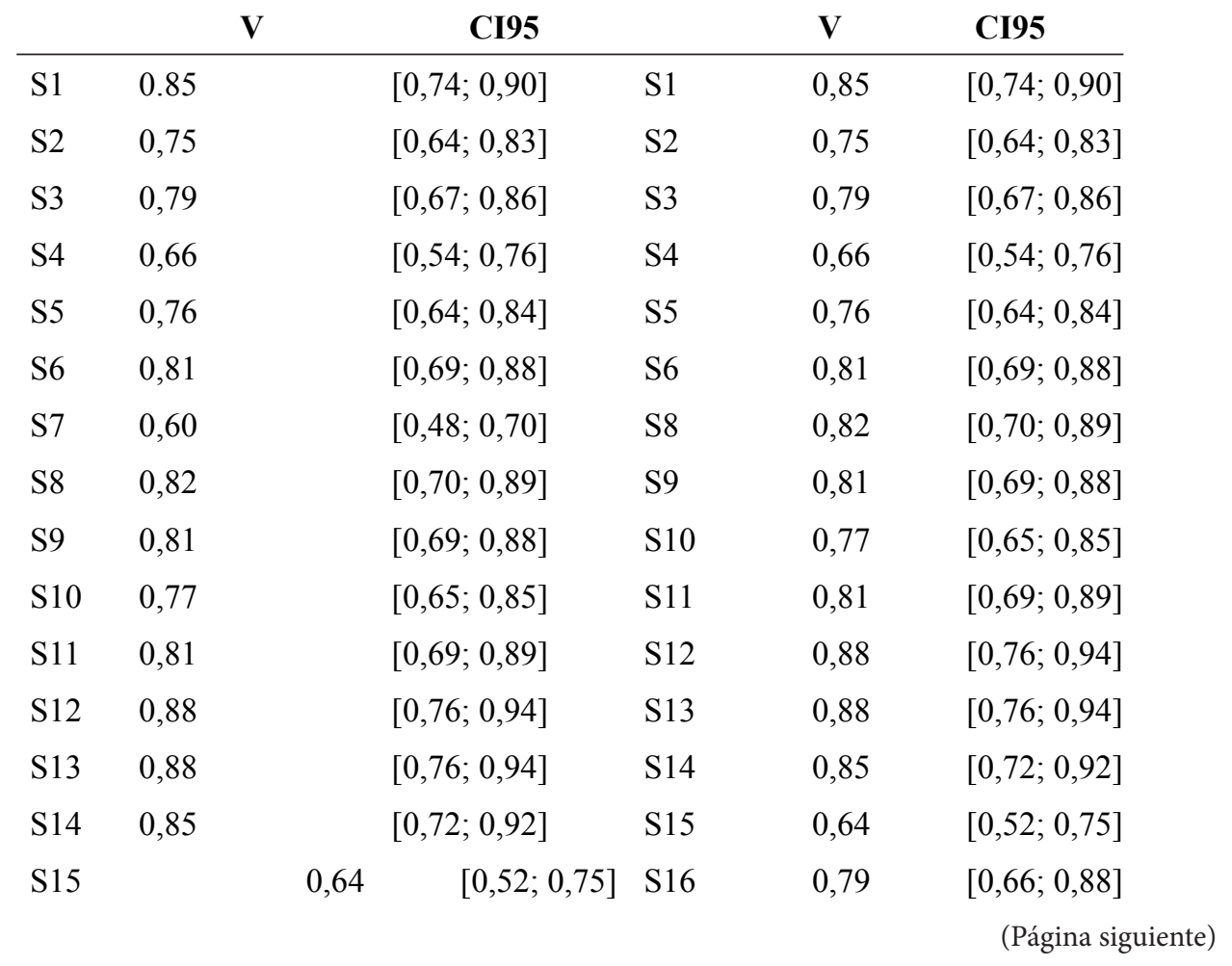


Tabla 3. Validación cualitativa por 13 jueces del instrumento CPDI en su versión 24 ítems (original) y Versión modificada de 23 ítems.

\begin{tabular}{|c|c|c|c|c|c|}
\hline & \multicolumn{2}{|c|}{ CPDI Original, 24 items } & \multicolumn{3}{|c|}{ CPDI modificada, 23 items } \\
\hline & $\mathbf{V}$ & CI95 & & $\mathbf{V}$ & CI95 \\
\hline S16 & 0,79 & {$[0,66 ; 0,88]$} & S17 & 0,81 & {$[0,68 ; 0,89]$} \\
\hline S17 & 0,81 & {$[0,68 ; 0,89]$} & S20 & 0,66 & {$[0,53 ; 0,77]$} \\
\hline S20 & 0,66 & {$[0,53 ; 0,77]$} & $\mathrm{S} 21$ & 0,84 & {$[0,71 ; 0,92]$} \\
\hline S21 & 0,84 & {$[0,71 ; 0,92]$} & S18 & 0,84 & {$[0,71 ; 0,92]$} \\
\hline S18 & 0,84 & {$[0,71 ; 0,92]$} & S19 & 0,77 & {$[0,64 ; 0,87]$} \\
\hline S19 & 0,77 & {$[0,64 ; 0,87]$} & S22 & 0,89 & {$[0,77 ; 0,97]$} \\
\hline S22 & 0,89 & {$[0,77 ; 0,97]$} & S23 & 0,87 & {$[0,74 ; 0,94]$} \\
\hline S23 & 0,87 & {$[0,74 ; 0,94]$} & S24 & 0,75 & {$[0,61 ; 0,85]$} \\
\hline S24 & 0,75 & {$[0,61 ; 0,85]$} & & & \\
\hline D1 & 0,76 & {$[0,62 ; 0,86]$} & D1 & 0,76 & {$[0,62 ; 0,86]$} \\
\hline D2 & 0,78 & {$[0,64 ; 0,88]$} & D2 & 0,81 & {$[0,68 ; 0,91]$} \\
\hline D3 & 0,78 & {$[0,64 ; 0,88]$} & D3 & 0,78 & {$[0,64 ; 0,88]$} \\
\hline D4 & 0,82 & {$[0,68 ; 0,92]$} & D4 & 0,82 & {$[0,68 ; 0,92]$} \\
\hline Total & 0,79 & {$[0,64 ; 0,89]$} & Total & 0,79 & {$[0,65 ; 0,90]$} \\
\hline
\end{tabular}

Debido a que la hipótesis nula para el índice de validez de contenido se cumplió para el ítem S7, se decidió descartarlo. De esta forma se mejoró los valores para la dimensión correspondiente. En este caso, el $\mathrm{V}_{\text {тот }}$ para la modificación del CPDI permanecieron igual. Dada la independencia entre los ítems, la eliminación del ítem S7 no afectó en absoluto los intervalos de confianza de los otros ítems (Tabla 3).

\section{DISCUSIÓN}

Los resultados de este estudio indican que el instrumento CPDI traducido al castellano muestra propiedades psicométricas relacionadas al contenido del instrumento que permitirán aplicar este instrumento en un estudio piloto para evaluar la confiabilidad y la validez de constructo en una muestra de la población peruana.

Específicamente, el instrumento en general muestra altos valores de validez por contenido calculados utilizando la metodología de Aiken, como se puede apreciar en la tabla 3. Sin embargo, se puede observar, que el ítem 7 (S7) tuvo que ser removido por no presentar valores significativos para el índice de validez de contenido. El retiro del S7 del CPDI original y la posterior modificación a 23 ítems no mostraron cambios en los valores significativos del $\mathrm{V}_{\text {ТОт }}$, como se puede observar en la Tabla 3. Esto es, porque el ítem S7 es consistente con lo que busca medir pero no mide necesariamente lo que el CPDI busca medir (distrés asociado al COVID-19, impacto emocional relacionado a COVID-19). Adicionalmente, es importante recordar los diferentes síntomas producidos por estrés asociado al evento, como por ejemplo: dificultad para conciliar el sueño, falta de concentración, irritabilidad, hiperreactividad, agitación psicomotriz, así como diversos síntomas relacionados a ansiedad y depresión (16). No obstante, los criterios clínicos actuales no sugieren que la "pérdida de la fe en las personas" sea un criterio indispensable para evaluar distrés relacionado a un evento, en este caso COVID-19. En todo caso, la "pérdida de la fe en las personas" es un concepto muy amplio que pudiese evaluar otras patologías o condiciones mentales (p.ej. esquizofrenia paranoide). Al modificarse el CPDI, se vio que a pesar de que los valores de las diferentes propiedades psicométricas disminuyeron, estos no disminuyeron su significancia ni disminuyeron abruptamente como para perder validez. Por lo tanto, se sugiere que en futuros estudios cuantitativos se compare las propiedades psicometricas de la escala modificada de 23 ítems versus la escala original de 24 ítems para la evaluación del impacto psicológico y emocional causado por COVID-19. 
En conclusión, traducido y adaptado el instrumento, así como evaluado por los expertos con respecto a su validez de contenido, se puede decir que el CPDI es adecuado para aplicarse en la población peruana. Queda pendiente, finalmente, aplicar el instrumento en poblaciones a manera de estudio cuantiativo, para determinar propiedades de la validez de constructo, como la estructura factorial, sensibilidad y especificidad para IE con COVID-19. Además, los autores de este estudio sugieren en posteriores estudios cuantiativos la comparación de la versión original con la modificada con 23 ítems. Esta última, incluye los ítems con mayor validez de contenido para evaluar el impacto emocional relacionado a COVID-19.

\section{Agradecimientos}

Los autores de este estudio quisieran agradecer a la Dra. Jianyin Qiu, quien proporcionó la versión inglesa del instrumento y la autorización para su traducción y uso para fines de investigación. Dicha autorización involucró la traducción, adaptación y validación del instrumento, así como su modificación.

Adicionalmente, los autores de este estudio desean agradecer a los siguientes doctores, que estuvieron involucrados en la evaluación del instrumento, tanto en su primera etapa (adaptación cultural) como en su segunda etapa (validación por jueces): Aitor Castillo, Lizardo Cruzado, Santiago Marquez, Miguel Sanchez, Galia Rojas, Favio Vega, Franz Alvarado, Marco Macavilca, Jose Luis Rondon, Mike Kabar, Nageli Lazo, Daniel Romero, Veronica Nué y Gabriela Gonzales

\section{Correspondencia:}

Bruno Pedraz-Petrozzi.

Klinikstrasse 36, D-35392, Giessen, Alemania.

Telf: (+49) 6419945773.

Correo electrónico: bruno.pedraz@med.uni-giessen.de

\section{REFERENCIAS BIBLIOGRÁFICAS}

1. Zhou F, Yu T, Du R, Fan G, Liu Y, Liu Z, et al. Clinical course and risk factors for mortality of adult inpatients with COVID-19 in Wuhan, China: a retrospective cohort study. Lancet. 2020;395(10229):1054-62.

2. Ministerio de Salud. Decreto Supremo No 008-2020SA. Lima: Ministerio de Salud; 2020. Disponible en: https://www.gob.pe/institucion/minsa/normaslegales/459902-008-2020-sa 09/04/2020
3. Presidencia del Consejo de Ministros. Decreto Supremo N ${ }^{\circ}$ 044-2020-PCM. Lima: Presidencia del Consejo de Ministros; 2020. Disponible en: https:// www.gob.pe/institucion/ $\mathrm{pcm} / \mathrm{n}$ ormas legales/460472-044-2020-pcm 09/04/2020

4. El País. Perú restringe la salida a la calle por género Madrid: El Pais; 2020. Disponible en: https://elpais. com/internacional/2020-04-04/peru-restringe-lasalida-a-la-calle-por-genero.html09/04/2020

5. Centros para el Control y la prevención de enfermedades. Salud mental y cómo sobrellevar la situación durante el COVID-19. Lima: Centros para el Control y la prevención de enfermedades; 2019. Disponible en: https://espanol.cdc.gov/enes/ coronavirus/2019-ncov/daily-life-coping/managingstress-anxiety.html 09/04/2020

6. Qiu J, Shen B, Zhao M, Wang Z, Xie B, Xu Y. A nationwide survey of psychological distress among Chinese people in the COVID-19 epidemic: implications and policy recommendations. Gen Psychiatry. 2020;33(2):e100213.

7. Afshar-Jahanshahi A, Mokhtari-Dinani M, NazarianMadavani A, Li J, Zhang SX. The distress of Iranian adults during the Covid-19 pandemic - More distressed than the Chinese and with different predictors. Brain Behav Immun. 2020.doi: 10.1016/j. bbi.2020.04.081

8. Zhang S, Yifei W, AfsharJahanshahi A, Jia J, HaenselSchmitt V. First study on mental distress in Brazil during the COVID-19 crisis. medR. 2020;(11).

9. Ministerio de Salud. Resolución Ministerial $\mathrm{N}^{\circ}$ 180-2020-MINSA. Lima: Ministerio de Salud; 2020. Disponible en: https://www.gob.pe/institucion/minsa/ normas-legales/473129-180-2020-minsa09/04/2020

10. de-Souza AC, Alexandre NMC, Guirardello E, de Souza ACe, Alexandre NMC, Guirardello E. Propriedades psicométricas na avaliação de instrumentos: avaliação da confiabilidade e da validade. Epidemiol e ServiçosSaúde. 2017;26(3):649-59.

11. Aiken LR. Three Coefficients for Analyzing the Reliability and Validity of Ratings. Educ Psychol Meas. 1985;45(1):131-42.

12. Penfield RD, Giacobbi PR. Applying a Score Confidence Interval to Aiken's Item ContentRelevance Index. Meas Phys Educ Exerc Sci. 2004;8(4):213-25.

13. Escurra LM. Cuantificación de la validez de contenido por criterio de jueces. Rev Psicol. 1988. Disponible en: https://www.researchgate.net/publication/270339 510_Cuantificacion_de_la_validez_de_contenido_ por_criterio_de_jueces $09 / \overline{0} / 2020$

14. Merino-Soto C, Livia Segovia J. Intervalos de confianza asimétricos para el índice la validez de 
Validación por expertos del Índice de Distrés Peri-traumático relacionado con la Enfermedad por Coronavirus 2019.

contenido: un programa Visual Basic para la $\mathrm{V}$ de Aiken. An Psicol. 2009; Disponible en: https://dialnet. unirioja.es/servlet/articulo? codigo $=2973711$ 09/04/2020

15. Aiken LR. Three coefficients for analyzing the reliability and validity of ratings. Educ Psychol Meas. 1985;45(1):131-42.
16. World Health Organization. The ICD-10 Classification of Mental and Behavioural Disorders: Diagnostic Criteria for Research. Ginebra: World Health Organization;1993.

Recibido: 08/05/2020

Aceptado: 05/12/2020 